\title{
Efecto de la administración de melatonina sobre la actividad de la ciclooxigenasa-2, la concentración sérica de metabolitos del óxido nítrico, los lipoperóxidos y la actividad de la glutatión peroxidasa en pacientes con enfermedad de Parkinson
}

Genaro Gabriel Ortiz1, E.W. Moráles-Sánchez², F.P. Pacheco-Moisés³, F.J. Jiménez-Gil4, M.A. MacíasIslas ${ }^{5}$, M.A. Mireles-Ramírez ${ }^{4}$ y H. González-Usiglit

${ }^{1}$ Laboratorio de Desarrollo-Envejecimiento, Enfermedades Neurodegenerativas, División de Neurociencias, Centro de Investigación Biomédica de Occidente, Instituto Mexicano del Seguro Social; ${ }^{2}$ Centro Universitario de Tonalá, Universidad de Guadalajara; ${ }^{3}$ Centro Universitario de Ciencias Exactas e Ingenierías, Departamento de Química, Universidad de Guadalajara; ${ }^{4}$ Unidad Médica de Alta Especialidad-Hospital de Especialidades, Centro Médico Nacional de Occidente, Departamento de Neurología, Instituto Mexicano del Seguro Social; ${ }^{5}$ Centro Universitario de Ciencias de la Salud, Departamento de Neurociencias, Universidad de Guadalajara, Guadalajara, Jalisco, México

\section{Resumen}

Objetivo: Determinar el efecto de la administración de melatonina (MEL) en la actividad de la ciclooxigenasa 2 (COX-2) y la concentración sérica de metabolitos del óxido nítrico, los lipoperóxidos y la actividad de la glutatión peroxidasa (GPx) en pacientes con enfermedad de Parkinson. Métodos: Ensayo piloto clínico aleatorizado longitudinal prospectivo y doble ciego. Se incluyeron 13 pacientes y se formaron dos grupos: MEL en dosis de 25 mg por vía oral cada 12 horas por 12 meses y placebo con almidón de maíz. Se evaluaron los pacientes mediante la Escala Unificada de la Enfermedad de Parkinson. Se les tomó una muestra de sangre al inicio y cada 3 meses hasta cumplir 12 meses. Resultados: la actividad de la COX-2 disminuyó, igual que los nitratos/nitritos (3, 6 y 9 meses) y los lipoperóxidos (9 y 12 meses); la GPx no mostró diferencias significativas.

PALABRAS CLAVE: Parkinson. Ciclooxigenasa 2. Nitratos-nitritos. Malondialdehído. 4-OH-alquenos. Glutatión peroxidasa.

\begin{abstract}
Objective: To determine the effect of melatonin (MEL) administration on ciclooxigenase 2 (COX-2) activity and serum concentration of nitric oxide metabolites, lipoperoxides and glutathione peroxidase (GPX) activity in patients with Parkinson's disease. Methods: Prospective double-blind randomized clinical pilot trial. 13 patients were included and two groups were formed: MEL at doses of $25 \mathrm{mg}$ orally every 12 hours for 12 months and placebo with corn starch. Patients were assessed using the Unified Parkinson's Disease Scale. A blood sample was taken at baseline and every 3 months until 12 months. Results: COX2 activity decreased as did nitrates/nitrites (3, 6 and 9 months) and lipoperoxides (9 and 12 months); GPx exhibited no significant differences.
\end{abstract}

KEY WORDS: Parkinson. Ciclooxigenase 2. Nitrates/nitrites. Malon-dialdehyde. 4-OH-alkenes. Glutathione peroxidase.

\author{
Correspondencia: \\ G. Gabriel Ortiz \\ Sierra Mojada, 800 \\ C.P. 44340, Guadalajara, Jal., México \\ E-mail: genarogabriel@yahoo.com
}

Fecha de recepción: 07-08-2017

Fecha de aceptación: 07-09-2017

DOI://dx.doi.org/10.24875/GMM.M000008
Gac Med Mex. 2017;153;Sup 2:S72-S81

Contents available at PubMed

www.anmm.org.mx 


\section{Introducción}

La enfermedad de Parkinson (EP) es una enfermedad neurodegenerativa que afecta al $1 \%$ de la población mayor de 65 años; es la segunda enfermedad neurodegenerativa de importancia, solo por debajo de la enfermedad de Alzheimer ${ }^{1}$. Descrita en 1817 por el médico inglés James Parkinson en su trabajo titulado An essay on the shaking palsy, la señala como una condición consistente en movimientos temblorosos involuntarios o parálisis agitante ${ }^{2}$. Jean-Martin Charcot, a finales del siglo xix, denominó EP a la «parálisis agitante» en honor de quien primero la describió. Tretiakoff descubrió la asociación entre la enfermedad y anomalías en la sustancia nigra, y posteriormente se demostró una disminución muy importante de la dopamina ${ }^{2,3}$

La etiología no está descrita completamente, pero la enfermedad es causada por la degeneración selectiva de neuronas dopaminérgicas de la sustancia nigra pars compacta (SNc) en la porción ventral del cerebro medio, que interrumpe la vía nigroestriatal, caracterizada por la presencia de inclusiones interneuronales conocidas como cuerpos de Lewy ${ }^{1,4,5}$. Los primeros signos y síntomas clínicos aparecen solo tras la pérdida de un $50-70 \%$ de las neuronas dopaminérgicas de la SNc. Así mismo, el medio ambiente desempeña un papel muy importante ${ }^{4}$. Se considera una enfermedad multifactorial que implica factores genéticos, ambientales y toxicológicos, y se ha asociado con procesos de óxido-reducción (especias reactivas de oxígeno [ROS]). Las principales manifestaciones clínicas son rigidez, temblor, bradicinesia, inestabilidad postural, alteraciones en la marcha, el olfato y la memoria, y demencia ${ }^{1,2,6}$.

La incidencia (mundial) de la EP es de 18 nuevos casos por cada 100,000 habitantes. Según los estudios auspiciados por el grupo de estudio Europarkinson, la prevalencia de la EP en la Unión Europea es del $1.43 \%$, y en los EE.UU. es del $1 \%$ en las personas de más de 60 años, y esta aumenta con la edad. La prevalencia relacionada con la edad es marcada en países que cuentan con distintos registros. En países como México no tenemos cifras confiables de incidencia y prevalencia. El Instituto Mexicano del Seguro Social (IMSS) contaba en 2016 con 62,161,705 derechohabientes, de los cuales 3,822,188 corresponden al Estado de Jalisco; tomando en cuenta la pirámide poblacional y su comportamiento futuro, la EP se convierte en una enfermedad de gran interés y problema de salud epidemiológico ${ }^{7,8}$.

Los procesos ambientales asociados con la EP no se han descrito por completo, pero se han relacionado los pesticidas y los herbicidas con el aumento en el riesgo de padecer la enfermedad, así como los metales pesados como el magnesio, el hierro, el zinc, el cobre y el aluminio9,10. Así mismo, existe evidencia que indica que el daño por estrés oxidativo se involucra en los padecimientos neurodegenerativos, entre ellos la EP. Productos modificados por la oxidación de ácidos nucleicos, proteínas, así como lipoperoxidación y oxidación de azúcares por medio del metabolismo, se han encontrado y correlacionado en lesiones del sistema nervioso central relacionadas con esta patología ${ }^{10}$. Una hipótesis sobre la presentación temprana de la EP debida a factores externos tóxicos se basa en el descubrimiento de tres inhibidores del complejo mitocondrial I: el paraquat, la rotenona y la 1-metil-4-fenil-1,2,3,6-tetrahidropiridina (MPTP), los cuales producen parkinsonismo ya que inhiben la cadena respiratoria mitocondrial con muerte selectiva de las neuronas dopaminérgicas $^{11,12}$. La disfunción mitocondrial debida a la inhibición del complejo I trae como consecuencia una depresión de la producción de ATP y la disfunción del proceso respiratorio mitocondrial, con un aumento del estrés oxidativo y de los productos secundarios de la lipoperoxidación, como el malondialdehído (MDA) y los 4-OH-alkenos (4-OHA), los productos metabólicos del óxido nítrico (NO) (nitratos/nitritos) y la activación de la ciclooxigenasa-2 (COX-2) que desencadena por varias vías el proceso de inflamación. Esta neuroinflamación, aunado a la muerte por estrés oxidativo, la interacción de las proteínas y la formación de cuerpos de Lewy, nos da por consiguiente una degeneración y la progresión de la $E P^{13}$. Las alteraciones por ROS producen errores de transcripción que ocasionan disfuncionalidad de la expresión de diferentes proteínas, como la $\alpha$-sinucleína, la parkina y la ubiquitina hidrolasa C-terminal, las cuales están relacionadas de manera directa en la EP.

El estrés oxidativo es producto (no único) de la cadena respiratoria mitocondrial debido a la generación de ROS y especies reactivas de nitrógeno (RNS), que en altas cantidades producen daño al sistema mitocondrial y a la célula, en especial la célula nerviosa, donde inducen señalización para la apoptosis ${ }^{11,14}$, así como oxidación de las membranas tanto neuronales como mitocondriales. Experimentos realizados en 
modelos animales, en los que se utiliza MPTP para provocar la muerte selectiva en neuronas dopaminérgicas en la SNc, han demostrado ser muy útiles para ejemplificar la muerte neuronal por reactivos altamente oxidantes que desencadenan estrés oxidativo y degeneran las neuronasde la región $\mathrm{SNc}^{11,13}$.

La melatonina (MEL) es una indol-amina producida por diversos organismos, desde las algas hasta los humanos. Tiene una evolución paralela al metabolismo aeróbico, y en los humanos es sintetizada y secretada por la glándula pineal, la retina, el tracto intestinal, la piel, los linfocitos, el timo y la médula ósea. Presenta un ritmo pulsátil en su producción y su distribución a lo largo del día y la noche, y durante la vida (reloj y calendario); disminuye con la edad, y por lo tanto la cantidad de MEL es menor en la ve$\mathrm{jez}^{15,16}$. La MEL realiza diversas funciones antioxidantes en la neurona, como la de barredor (scavenger), donde disminuye el daño neuronal ya que interviene en procesos de neuroinflamación, y disminuye la producción de ROS y RNS, así como los productos de la lipoperoxidación de las membranas ${ }^{17-19}$.

Las ciclooxigenasas son enzimas que poseen una doble actividad: una ciclooxigenasa y otra peroxidasa. Como ciclooxigenasas catalizan la bis-oxigenación del ácido araquidónico, el cual se transforma en un hidroperóxido que genera la prostaglandina $G_{2}$ $\left(P G G_{2}\right)$, y como peroxidasa cataliza la reducción de la $P G G_{2}$. La activación de la COX-2 puede llevarse a cabo por un activador hidroperóxido en las células inflamatorias del SNC, que se forma del acoplamiento del anión superóxido $\left(\mathrm{O}_{2}^{-}\right)$y el $\mathrm{NO}$, denominado ácido peroxinitroso $(\mathrm{ONOOH})$.

Con el aumento de la esperanza de vida y el cambio generacional, la EP se vuelve no solo un problema de salud, sino también un reto en el tratamiento y la atención hacia estos pacientes. Este proceso, además de ser costoso para los servicios de salud, tiene como consecuencia la aceleración de las manifestaciones clínicas, las cuales se vuelven devastadoras para los pacientes y sus familias. En el campo de las enfermedades neurodegenerativas, las diversas terapias existentes en ocasiones solo limitan la actividad de la enfermedad. La suma de diversas terapias podría dar como resultado un verdadero retardo de la evolución de la EP, una mejoría o una mejor calidad de vida para los pacientes.

En trabajos previos en nuestro laboratorio hemos demostrado la actividad antioxidante de la MEL en modelos experimentales de EP (con MPTP), en especial su actividad antioxidante, y así mismo hemos demostrado la inhibición de la actividad COX-2, lo que nos lleva a pensar que la suplementación de MEL a los pacientes con EP podría ser de utilidad en el tratamiento de las manifestaciones clínicas o para retrasar la progresión de la enfermedad ${ }^{9-14}$. El objetivo de este trabajo fue evaluar el efecto de la MEL en la actividad de la COX-2, la concentración sérica de metabolitos del NO (nitratos/nitritos), los lipoperóxidos (MDA y 4-OHA) y la actividad de la glutatión peroxidasa (Gpx) en pacientes con EP.

\section{Métodos}

Se llevó a cabo un ensayo-piloto clínico aleatorizado, longitudinal, prospectivo y doble ciego. El universo de estudio fueron los pacientes con diagnóstico de EP derechohabientes del IMSS atendidos en la Clínica de Trastornos del Movimiento de la UMAE-HE del Centro Médico Nacional de Occidente, de Guadalajara, Jalisco, México.

Se formaron dos grupos: a uno (grupo MEL) se le suplementó con $50 \mathrm{mg}$ de MEL en dosis de $25 \mathrm{mg}$ por vía oral cada 12 horas por 12 meses, y al grupo placebo (grupo CON) se le administró almidón de maíz; a ambos grupos se les realizó la evaluación inicial del estado del paciente mediante la Escala de Evaluación Unificada de la Enfermedad de Parkinson (UPDRS, Unified Parkinson Disease Rating Scale), así como toma de muestra de sangre en la visita inicial y consecutivamente cada 3 meses hasta cumplir 1 año de intervención. Al inicio del manejo y el establecimiento del correcto diagnóstico fue necesario dar vigilancia y seguimiento, así como cumplir con el perfil de bioseguridad (pruebas generales de bioquímica clínica) para tener certidumbre de que no existían efectos secundarios. El proceso de selección y seguimiento fue el siguiente:

- Historia y valoración clínica, con la cual se determina cuál es el padecimiento real

- Una vez que se hace el diagnóstico adecuado de EP se inicia su manejo farmacológico, al que se da seguimiento por 1 mes para determinar si es el adecuado.

- Evaluación para corroborar las respuestas adecuadas o inadecuadas al tratamiento, mejora sintomática y ajuste de dosis; se vigila por 2 meses más para determinar cuál será su manejo o si requiere un nuevo ajuste.

- Una vez establecido el tratamiento y su alcance, se invita al paciente para la participación en el proyecto de investigación. 
Existen diferentes esquemas farmacológicos para el tratamiento de la EP; se selecciona el más adecuado a cada caso y homogéneo para cada grupo. A partir de este momento se puede incluir el paciente. Una vez que el paciente decide participar, se realiza estadificación mediante la escala de Hoehn y Yarh.

Se realizó historia clínica y aplicación de UPDRS, que se repite a los 12 meses después de la última toma del suplemento o placebo. Se obtienen muestras de sangre $(15 \mathrm{ml})$ al inicio y a los 3, 6, 9 y 12 meses. De ellas se separó suero para poder realizar las determinaciones. Tras extraer el suero, este se congeló a $-80{ }^{\circ} \mathrm{C}$ para su adecuado resguardo. Las muestras fueron procesadas para la determinación de la actividad de COX-2, así como de nitratos, nitritos, 4-OHA, MDA y GPx.

La actividad de COX-2 fue determinada mediante la utilización del paquete COX activity assay kit número de catálogo 760151 de Cayman Chemical Company, a partir de las muestras obtenidas, las cuales fueron procesadas siguiendo los pasos recomendados por el paquete, el cual se basa en la utilización de un ensayo colorimétrico que se lee con un espectrofotómetro a $590 \mathrm{~nm}$ para determinar la absorbancia y obtener los resultados de la actividad de la COX-2. El principio de la medición consiste en el análisis de la muestra (en este caso suero), a la cual se le agrega un inhibidor de COX-2 y posteriormente ácido araquidónico, que inicia la reacción; luego se midió a $590 \mathrm{~nm}$ y se determinaron los resultados.

Los lipoperóxidos se descomponen en derivados del carbonilo: MDA y 4-OHA. Esta medición se basa en la reacción del cromógeno $\mathrm{N}$-metil-2-fenilindol con el MDA y los $4-\mathrm{HOA}$ a $45^{\circ} \mathrm{C}$. En este ensayo, una molécula de MDA o 4-HOA reacciona con dos moléculas de N-metil-2-fenilindol para producir un cromóforo estable con una absorbancia máxima a $586 \mathrm{~nm}$. La producción de MDA y 4-OHA se utilizó como marcador de daño oxidativo, y por tal motivo se utilizó el paquete Kit LPO-586, el cual tiene ventajas sobre otros porque el agente cromógeno reacciona con los productos de lipoperoxidación a $45^{\circ} \mathrm{C}$. Esta reacción dio como resultado un producto estable, el cual tiene un límite máximo de absorbancia a $585 \mathrm{~nm}$ de longitud de onda, así como una baja temperatura de incubación que elimina los artefactos no deseables.

El NO se cuantificó de forma indirecta, ya que su metabolismo forma nitritos/nitratos y al cuantificar estos medimos la concentración de NO. Se utilizó para este ensayo un paquete comercial (Calbiochem Nitric Oxide Assay Kit colorimetric). Las muestras de cada experimento fueron obtenidas a partir de suero de los pacientes, y fueron almacenadas a $-80{ }^{\circ} \mathrm{C}$ hasta el momento de realizar su análisis. Posteriormente se realizó la desproteinización del suero, donde los nitritos y los nitratos fueron medidos. Los nitratos son convertidos a nitritos por medio de la incubación con nitrato reductasa en presencia de NADPH; posteriormente se utilizó lactato deshidrogenasa para eliminar el NADPH. Volúmenes iguales de muestra y de reactivo de Griess fueron incubados a temperatura ambiente durante 10 minutos para posteriormente utilizar un espectrofotómetro y medir la absorbancia a $550 \mathrm{~nm}$. Al final, la concentración de NO (nitritos) fue determinada con una curva de nitrato de sodio como estándar. Todos los ensayos químicos se realizaron en un espectrofotómetro Perkin-Elmer EZ-50.

El presente trabajo cumplió con toda la normatividad y respetó todos los tratados de bioética. Se realizó previa cita y explicación de los posibles resultados, y mediante explicación de los procedimientos a realizar, con la autorización por parte del paciente mediante firma de carta de consentimiento informado. Fue evaluado y aceptado por el comité de investigación del Instituto Mexicano del Seguro Social bajo el registro R-20101301-9.

La captura de datos y el análisis estadístico se realizaron en el programa SPSS (v20windows). Se obtuvieron datos generales de los pacientes, medidas de tendencia central (media) y medida de dispersión, para ver cuánto tienden a alejarse los valores concretos del promedio. También utilizamos estadística descriptiva, análisis vivariado entre el grupo control y el grupo experimental, donde se consideró estadísticamente significativa una $p \leq 0.05$. Los resultados se muestran en gráficas y tablas.

\section{Resultados}

Tras la selección de los pacientes y el desarrollo del proyecto se concluyó el mismo con la inclusión de 13 pacientes: seis en el grupo MEL y siete en el grupo CON. Posterior a las determinaciones, se abrió el ciego para poder realizar el seguimiento correspondiente, así como el análisis estadístico.

\section{Actividad de la ciclooxigenasa 2}

La determinación de la actividad de la COX-II a lo largo de la intervención mostró, a diferencia de la basal, que los pacientes pertenecientes al grupo MEL poseían menor actividad en comparación con los del grupo 
CON; en especial, estos mostraron una menor actividad a los 3 y 12 meses. Los análisis comparativos entre los grupos de la actividad de la COX-2 al inicio y al final mostraron resultados importantes sobre la determinación de esta actividad. El análisis estadístico mostró que la comparación del grupo MEL y el grupo CON al final de la intervención fue significativa, con $p \leq 0.0313$ (Fig. 1), con menor actividad en el grupo MEL.

\section{Óxido nítrico}

Los resultados se muestran en la figura 2. Tanto en el grupo MEL como en el grupo COM no se encuentran diferencias significativas a los tiempos basal y 3 meses $(37.6 \pm 3.3$ y $38.3 \pm 6.7$ en el grupo MEL, y $40.2 \pm 2.9$ y $40.9 \pm 2.9$ en el grupo CON). Posterior a esto, a los 6,9 y 12 meses apreciamos una disminución de la concentración de catabolitos del NO (nitratos/nitritos) en el grupo MEL $(34.7 \pm 4.3,35.7 \pm$ 1.6 y $33.2 \pm 1.2$, respectivamente). Estos resultados son significativos estadísticamente, en especial a los 12 meses de tratamiento $(p \leq 0.05)$.

\section{Lipoperóxidos}

Los resultados del comportamiento de los grupos MEL y CON se muestran en la figura 3, donde se observa que hubo fluctuaciones en ambos grupos. En el análisis apreciamos una disminución de los productos de lipoperoxidación (MDA y 4-OHA), con diferencias significativas principalmente en el grupo MEL a los 9 y 12 meses, en comparación con el grupo COM $(1.19 \pm 0.05$ y $1.2 \pm 0.03$ en el grupo MEL y 1.52 \pm 0.02 y $2.02 \pm 0.02$ en el grupo CON; $p \leq 0.05$ ).

\section{Glutatión peroxidasa}

Los resultados obtenidos en el análisis de la actividad de la enzima Gpx no mostraron ninguna diferencia al comparar los grupos MEL y CON en todos los tiempos estudiados (Tabla 1).

\section{Escala de Evaluación Unificada de la Enfermedad de Parkinson}

Los resultados de la aplicación de la UPDRS mostraron una tendencia a la baja de la puntuación, una disminución en los valores obtenidos en los pacientes que tuvieron MEL como tratamiento, y no en los de placebo. Para poder determinar si existía algún cambio se realizó el análisis estadístico de las diferencias de los puntajes de UPDRS inicial menos final, y las diferencias fueron analizadas de manera estadística para determinar la presencia de una diferencia, que fue significativa con $p=0.0226$. Aquellos pacientes de EP que conformaron el grupo MEL tuvieron una disminución en la puntuación de la escala, en comparación con el grupo CON, en quienes aumenta la puntuación de la escala al final de la intervención. La disminución de los valores evaluados en la escala

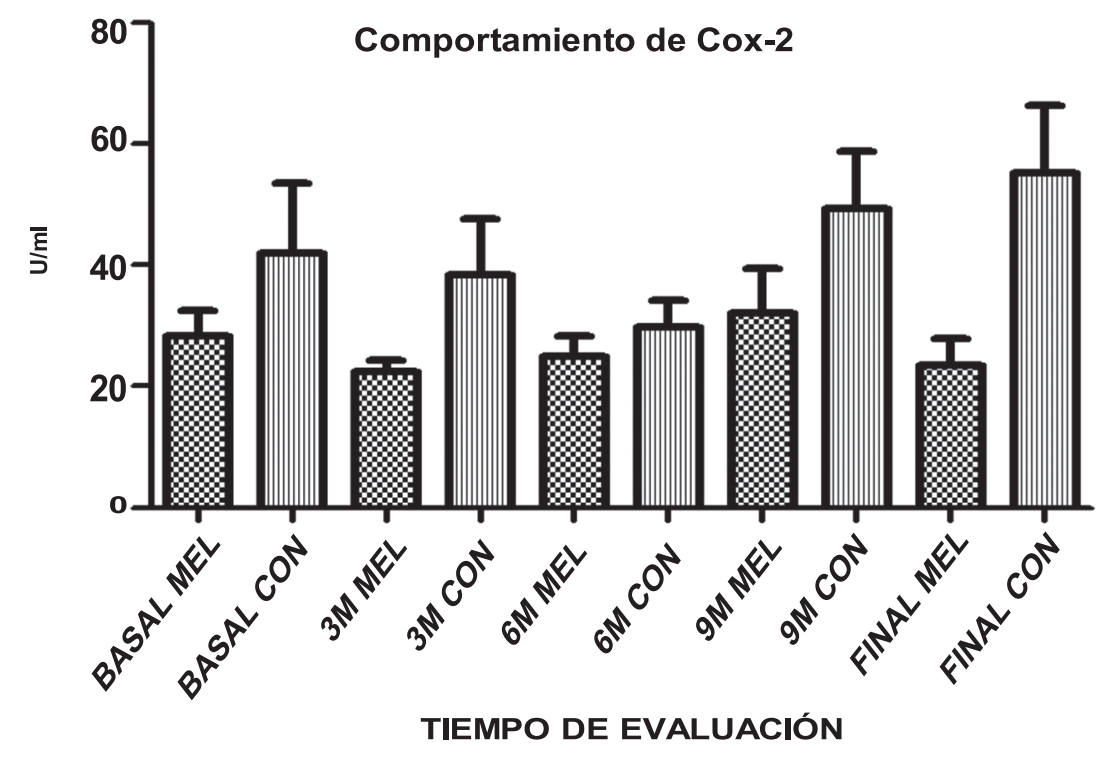

Figura 1. Actividad de la COX-2 a lo largo de la intervención y comparación de los grupos MEL y CON, con disminución de su actividad a los 9 y 12 meses de la administración de MEL en comparación con el grupo CON. 


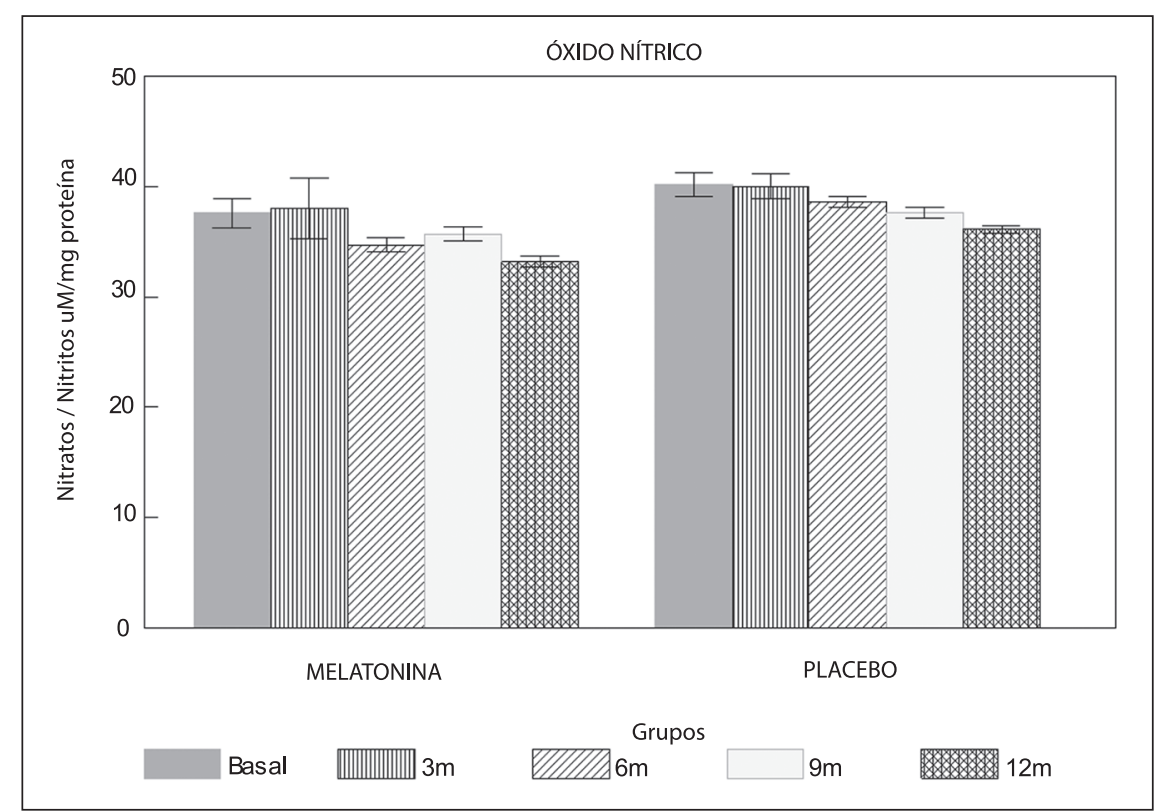

Figura 2. Resultados de la concentración de catabolitos del NO expresada en nitratos/nitritos. Los valores expuestos son la media $\pm D E$ de cada tiempo estudiado (basal, 3, 69 y 12 meses), tanto en el grupo suplementado con MEL como en el grupo CON. Estos resultados muestran una disminución de los catabolitos del NO en el grupo MEL respecto al grupo CON en los tiempos 6, 9 y 12 meses.

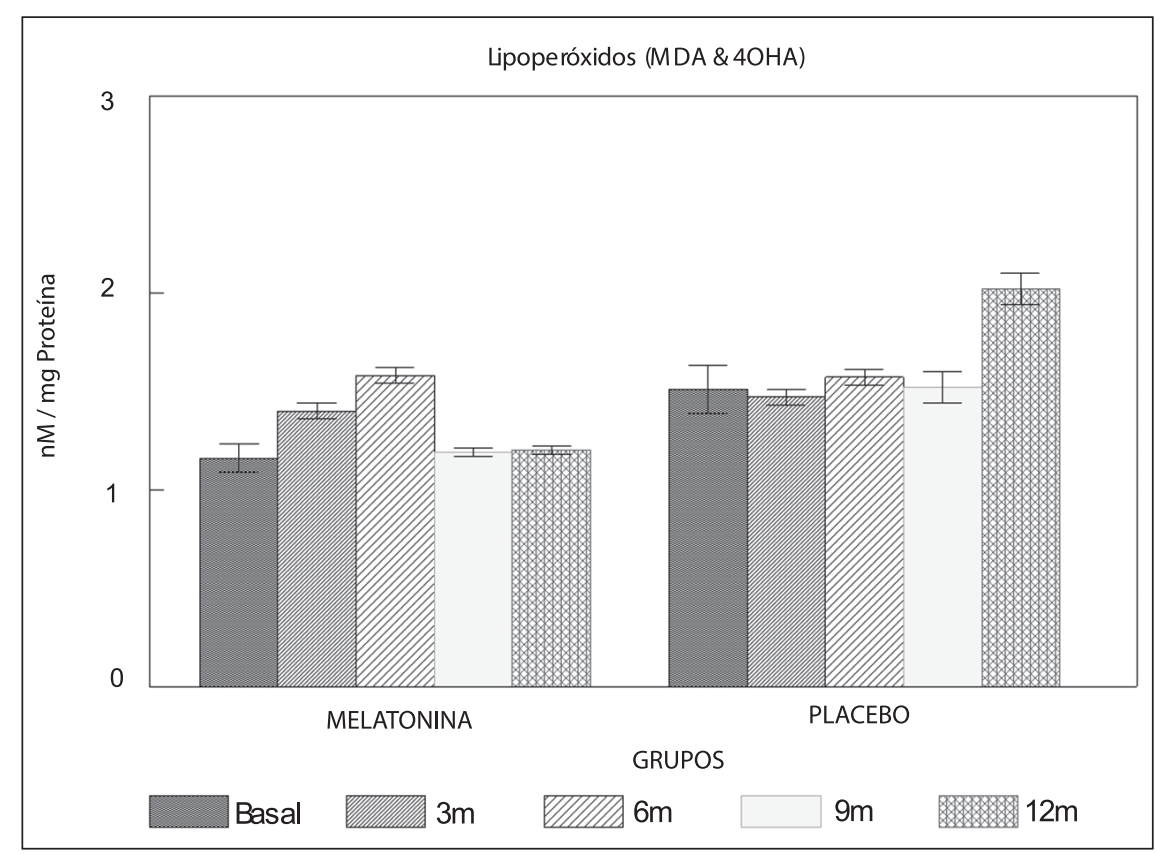

Figura 3. Resultados de la concentración de productos de la lipoperoxidación, MDA y 4-HOA, expresados en media \pm DE de cada tiempo estudiado (basal, 3, 69 y 12 meses), tanto en el grupo MEL como en el grupo CON. Estos resultados muestran una disminución de lipoperóxidos en el grupo MEL en comparación con el grupo CON en los tiempos 9 y 12 meses.

tras 1 año de la evaluación inicial no mostró un aumento en la progresión de la EP (Fig. 4).

\section{Discusión}

El empleo de MEL en el manejo de diversas enfermedades neurodegenerativas ha estado presente $y$ se han propuesto diversos modos de administración y diversas dosis. Cardinali, et al. ${ }^{16}$ en Argentina, propuso el consumo de dosis que van de los 3 a los $15 \mathrm{mg}$ para tratar los trastornos del sueño en los pacientes con EP. Pandi-Perumal, et al. ${ }^{15}$ la mencionan por su interacción en el sueño en pacientes con Alzheimer y EP. También el grupo de investigación a 


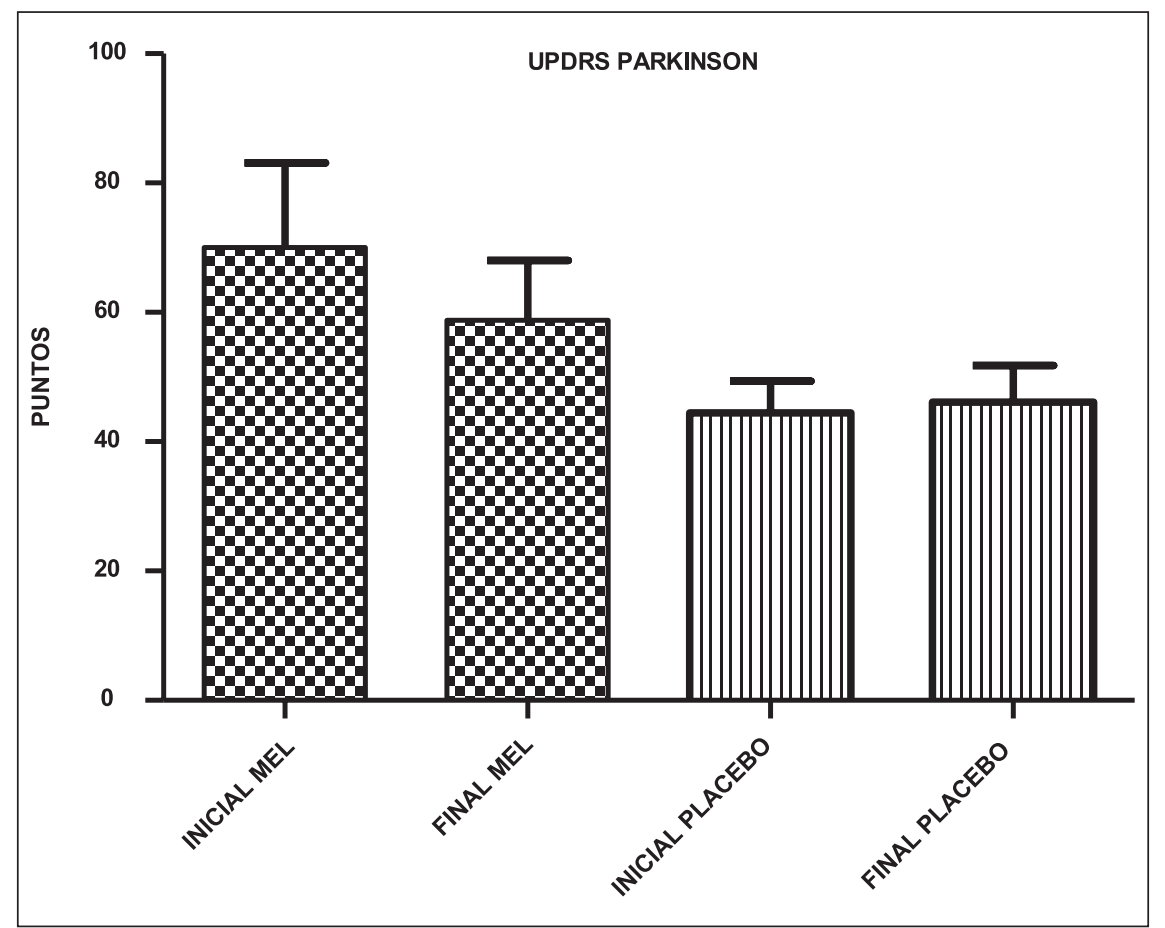

Figura 4. Comparación de las medias obtenidas de la valoración realizada a los grupos. La medición se realizó al inicio y al final de la intervención. El grupo que recibió MEL tuvo una tendencia a la disminución de la puntuación en la escala UPDRS.

Tabla 1. Resultados de la actividad de la Gpx en cada tiempo estudiado, tanto en el grupo MEL como en el grupo CON. Estos resultados no muestran diferencias significativas

\begin{tabular}{ccccccc}
\hline \multicolumn{3}{c}{ Melatonina } & & \multicolumn{3}{c}{ Placebo } \\
\cline { 1 - 3 } \cline { 5 - 6 } Media & DE & $\mathbf{p}=\mathbf{0 . 0 5}$ & & Media & DE & $\mathbf{p}=\mathbf{0 . 0 5}$ \\
\hline Basal & 1.03 & 0.013 & & 1.03 & 0.006 & 0.03 \\
$3 \mathrm{~m}$ & 1.03 & 0.012 & & 1.03 & 0.02 & 0.02 \\
$6 \mathrm{~m}$ & 1.04 & 0.015 & & 1.04 & 0.02 & 0.03 \\
$9 \mathrm{~m}$ & 1.05 & 0.018 & & 1.03 & 0.02 & 0.04 \\
$12 \mathrm{~m}$ & 1.04 & 0.015 & & 1.04 & 0.02 & 0.03 \\
\hline $\mathrm{n}=67$. & & & & &
\end{tabular}

cargo del Dr. Reiter menciona la MEL como un potente antioxidante para el manejo de enfermedades como el Alzheimer y la EP, y en colaboración con el Dr. Mayo propusieron a la MEL como un regulador de la actividad inflamatoria y antioxidante en la EP'1.

En una publicación de nuestro equipo de trabajo en el Laboratorio de Desarrollo y Envejecimiento del Centro de Investigación Biomédica de Occidente se analizan los resultados de la administración de MEL en el control de la COX-2 y las ROS en un modelo múrido de EP utilizando MPTP. En este trabajo se demostró una actividad disminuida de la COX-2 por acción de la $\mathrm{MEL}^{13}$. La interacción de la MEL en su forma reactiva de potente antioxidante frente a los productos del estrés oxidativo de la mitocondria desempeña un papel importante en la neuroinmunomodulación de los factores proinflamatorios a la muerte neuronal. Disminuye la activación de productos que, como la COX-2 favorecen un incremento en la inflamación y la cicatrización, y por consiguiente incrementan la progresión de la EP ${ }^{22}$. Los resultados obtenidos en este trabajo nos llevan a probar el posible uso terapéutico que puede tener y utilizar la MEL como coadyuvante para el manejo de la EP, ya que disminuyó la actividad de la COX-2.

Nuestro trabajo apoya la evidencia de la eficacia de la MEL como antioxidante y depurador de radicales libres, debido a su estructura química indólica y a un potencial redox elevado. La MEL cede electrones fácilmente, lo que hace que actúe como un potente agente reductor. La MEL depura el radical $\bullet \mathrm{OH}$ dando lugar a 3-OH-melatonina cíclica, que se excreta por la orina y puede emplearse como marcador del estrés oxidativo. Hoy día sabemos que la MEL regula la expresión de diversos genes que controlan la producción de una serie de proteínas, entre ellas las principales enzimas antioxidantes endógenas, como la GPx, la glutatión reductasa (GRd), la superóxido dismutasa (SOD) y la catalasa. Así mismo, la expresión de enzimas prooxidantes y proinflamatorias, como la NO sintasa inducible y la COX-2, están bajo regulación genómica de la MEL. La regulación de la MEL se hace 
en base a potenciar la expresión de los sistemas endógenos antioxidantes y reducir los proinflamatorios. Parece ser que existe una colaboración entre ambos tipos de receptores, nucleares y de membrana, en la regulación de estos sistemas enzimáticos.

\section{Enfermedad de parkinson, óxido nitrico (nitratos/nitritos) y melatonina}

En este estudio mostramos que la MEL tuvo un efecto antioxidante en la concentración de los metabolitos del NO (nitratos/nitritos), lo cual puede ser explicado de dos formas: la primera es que actúa como un eficiente antioxidante, ya que depura RNS tales como el anión peroxinitrito (ONOO-). Estos aniones presentan una alta toxicidad, similar a la del radical $\bullet \mathrm{OH}$, por lo que su eliminación por la MEL proporciona una defensa antioxidante más eficaz que el glutatión o las vitaminas antioxidantes, y la otra es su efecto sobre la enzima NO sintasa, tanto la constitutiva como la inducible. Este efecto se ve más efectivo en los tiempos 6, 9 y 12 meses de tratamiento (intervención); si bien observamos una menor tendencia desde los grupos iniciales, estos son estadísticamente significativos. Las evidencias experimentales apoyan desde un principio estos resultados. Una de las discrepancias que podemos encontrar en nuestros resultados es que en el grupo MEL es menor la concentración basal de nitratos y nitritos (NO), pero hay que recordar que este fue un estudio doble ciego aleatorizado en humanos, por lo que los grupos desde un inicio pueden salir distintos. No podemos tener un control, como lo hicimos en un trabajo experimental previo en modelo animal, pero las respuestas al tratamiento a los tiempos 6, 9 y 12 meses no nos dejan duda de su efecto en la concentración de NO (sus metabolitos). Por otra parte, hay que hacer obligada referencia a que la MEL ejerce algunas de sus acciones mediante su unión a la calmodulina (proteína fijadora de calcio). La importancia de la interacción MEL-calmodulina, la cual se ha relacionado con su efecto antiexcitotóxico, es que tras esta unión la MEL inhibe la NO sintasa neuronal ${ }^{23}$, así como los receptores nucleares del ácido retinoico $\beta$ RZR, la familia de receptores ROR $\alpha-1$ y ROR $\alpha-2^{24}$. Acciones antioxidantes de la MEL comprenden efectos dependientes e independientes del receptor, y en particular la modulación del metabolismo mitocondrial ${ }^{25}$. Este efecto es de vital importancia también en la interpretación de estos datos, ya que la calmodulina posee un efecto directo sobre la enzima NO sintasa en esta ruta: la isoforma constitutiva de la NO sintetasa, tanto en el endotelio como en las neuronas, se halla presente en forma inactiva hasta que se produzca un aumento del calcio intracelular. En las células endoteliales, los agonistas como la acetilcolina, el difosfato deadenosina, la bradicinina o el estrés oxidativo, actúan como estímulos aumentando el calcio intracelular. La calmodulina se une al calcio y el compuesto así formado, calmodulina-calcio, activa la NO sintasa, estimulando la producción de pequeñas cantidades de NO, hasta que disminuye la concentración de calcio. EI NO estimula la enzima guanilato ciclasa para que convierta el trifosfato cíclico de guanosina en monofosfato cíclico de guanosina, importante molécula moduladora y reguladora del metabolismo. La MEL regula la actividad de las enzimas antioxidantes GPx, GRd y SOD, manteniendo unas concentraciones adecuadas de glutatión dentro de la célula y de la mitocondria.

\section{La enfermedad de Parkinson, la lipoperoxidación y la melatonina}

Un estado de estrés oxidativo induce en la célula efectos tóxicos por oxidación de lípidos, lo cual produce acumulación de agregados intracelulares, disfunción mitocondrial, excitotoxicidad y apoptosis. Este daño oxidativo es común en las enfermedades neurodegenerativas y aún no está claro si contribuye iniciando el proceso o es una consecuencia del mismo. La formación de lípidos modificados por oxidación puede causar disfunción celular y, en células posmitóticas como las neuronas, la muerte o la apoptosis. La peroxidación de ácidos grasos poliinsaturados en las membranas celulares inicia un deterioro acumulativo de las funciones membranales y provoca disminución en la fluidez, reducción en el potencial electroquímico y aumento de la permeabilidad de la membrana.

El $\mathrm{HO}$ es un radical extremadamente reactivo capaz de abstraer un átomo de hidrógeno de los carbonos alílicos (=CH-) de una multitud de constituyentes celulares, entre ellos los ácidos grasos insaturados de las membranas celulares y las bases púricas y pirimídicas de los ácidos nucleicos. Los compuestos resultantes de la abstracción de hidrógeno son radicales libres alquilo; los radicales libres de este tipo de las bases de los ácidos nucleicos frecuentemente se dimerizan, como es el caso de los dímeros de timina. Los radicales alquilo derivados de los ácidos grasos insaturados son aptos para dar reacciones de adición con oxígeno molecular, generando radicales peroxilo (ROO) que a su vez, a través de una nueva 
abstracción de hidrógeno a otro carbono alílico de otro ácido graso insaturado, reacción favorecida por la estructura en bicapa lipídica de las membranas biológicas, produce un hidroperóxilo $(\mathrm{ROOH})$ y un nuevo radical alquilo, lo que establece una reacción de radicales libres en cadena. La peroxidación lipídica o lipoperoxidación es el proceso químico en el que las reacciones ocurren sobre ácidos grasos insaturados. Los grupos $-\mathrm{OOH}$ de los hidroperóxidos $(\mathrm{ROOH})$, localizados en carbonos terciarios en medio de la cadena hidrocarbonada de los ácidos grasos insaturados de las membranas biológicas, llevan a una distorsión de su espacio hidrófobo y a una pérdida de su función biológica. La acumulación de grupos hidroperóxido en carbonos alílicos lleva a su vez a la rotura de la cadena carbonada y a la formación de malonaldialdehído ( $\mathrm{COH}-\mathrm{CH} 2-\mathrm{CHO})$, que es un indicador de la presencia de lipoperoxidación. Las reacciones de terminación que pueden involucrar a radicales alquilo y peroxilo, cabe señalar que, a su vez, indican la formación de oxígeno singulete $\left(\mathrm{O}_{2}\right)$, el estado electrónico excitado del oxígeno molecular, como producto secundario y marcador del proceso de lipoperoxidación.

En la EP, las concentraciones de MDA (un marcador de oxidación de lípidos) y de 4-OHA están aumentadas. En esta enfermedad hay niveles altos de productos finales de la glucosilación, resultado de una oxidación alterada de glucosa y que causan oxidación irreversible de proteínas en la SNc y en la corteza cerebral. Otro factor que contribuye al daño oxidativo es que la distribución de metales de transición en el cerebro muestra grandes diferencias regionales. Las regiones con grandes cantidades de hierro $\left(\mathrm{Fe}^{++}\right.$, que es fácilmente oxidable) en la SNc tienen un riesgo de sufrir un ataque oxidativo más agresivo por lipoperóxidos. En nuestro estudio el tratamiento con MEL fue eficaz como antioxidante ya que redujo los niveles de los catabolitos del óxido nítrico y los lipoperóxidos en relación con los valores basales. Esto es más evidente a los 9 y 12 meses de tratamiento. En tanto que en el grupo control los valores de los lipoperóxidos son similares a los 3,6 y 9 meses del tratamiento en relación al basal. Aunque a los 12 meses dichos valores se incrementan de manera importante.

\section{La enfermedad de Parkinson, la actividad de la enzima glutatión peroxidasa y la melatonina}

Con el antecedente de que la MEL administrada exógenamente provoca un aumento de dos veces en la actividad GPx dentro de 30 minutos en el cerebro en el modelo experimental (MPTP), y sabiendo que la actividad cerebral de la GPx es mayor durante la noche que durante el día, estos datos se pueden correlacionar con las concentraciones de MEL en diferentes tejidos. La GPx es una de las enzimas involucradas en la eliminación de peróxidos en el cerebro. Por tanto, su actividad disminuye la probabilidad de que se produzca el radical hidroxilo a partir de la reacción de los peróxidos con metales de transición (vía de Fenton). Hoy en día sabemos que el radical hidroxilo es el radical más nocivo conocido. La inducción de la Gpx es un mecanismo importante por el cual la MEL ejerce sus efectos neuroprotectores potentes. Pero debemos ser honestos y mencionar que los ensayos terapéuticos con MEL han sido eficaces en el retraso de la progresión de la enfermedad de Alzheimer, pero no de la EP, lo que puede ser debido a varias causas: de método y farmacológicas. En los primeros estudios clínicos existen diferencias que no dejan clara la concentración de MEL administrada, los tiempos de administración, el tiempo del estudio, etc. Sabemos de estudios que mencionan que la administración de MEL causa un problema motor mayor, aunado al que ya presenta el paciente, lo que nos debe llevar a ser cautos. Solo un estudio sistemático y a largo plazo nos dará la razón. En este estudio (no olvidar que es un estudio piloto), con una muestra pequeña y en la cual no encontramos una actividad diferente de la Gpx en ambos grupos estudiados (MEL vs. COM), esto lo explicamos por el número de pacientes estudiados. La pregunta que queda pendiente por resolver es qué tan independiente es la respuesta de la GPx y el estrés oxidativo, así como la concentración de dopamina vs. MEL y el papel de todo el sistema redox: glutatión, Gpx, glutatión reductasa y uperóxido dismutasa.

\section{Escala de Evaluación Unificada de la Enfermedad de Parkinson}

Por último, para determinar la asociación entre esta variable y la UPDRS se realizó una prueba de correlación entre la actividad de la COX- 2 y el puntaje UPDRS. Al realizar el análisis se halló una asociación entre la actividad de la COX-2, el consumo de MEL y la disminución de los valores de UPDRS. Esta correlación no fue determinante, pero muestra una asociación entre los valores de la actividad de la COX-2 y la disminución del puntaje de la UPDRS de 0.4 (si el valor fuera 0 , no existiría asociación alguna entre las variables). 


\section{Agradecimiento}

\section{In memoriam Dr. Alfredo I. Feria Velasco.}

\section{Bibliografía}

1. Mayo JC, Sainz RM, Tan D-X, et al. Melatonin and parkinson's disease. Endocrine. 2005;27:169-78.

2. García S, López B, Dávalos EGM, et al. Breve reseña histórica de la enfermedad de Parkinson. De la descripción precipitada de la enfermedad en el siglo XIX, a los avances en biología molecular del padecimiento. Med Int Mex. 2010;26:350-73.

3. García S, Suárez SS, Dávalo EM, et al. Perspectiva histórica y aspectos epidemiológicos de la enfermedad de Parkinson. Med Int Mex. 2008;24:28-37.

4. Barzilai A, Melamed E. Molecular mechanisms of selective dopaminergic neuronal death in Parkinson's disease. Trends Mol Med. 2003;9:126-32.

5. Gandhi S, Wood NW. Molecular pathogenesis of Parkinson's disease. Hum Mol Genet. 2005;14(Supl 2):2749-55.

6. Hoehn MM, Yahr MD. Parkinsonism: onset, progression and mortality. Neurology. 1967; 17:427-42.

7. Bennett DA, Beckett LA, Murray AM, et al. Prevalence of parkinsonian signs and associated mortality in a community population of older people. N Engl J Med. 1996;334:71-6.

8. Instituto Nacional de Estadística y Geografía (INEGI). Disponible en http://www.inegi.org.mx/default.aspx. Consultado el 8-02-2017.

9. Ortiz GG, Moisés FPP, Macías-Islas MA, et al. Toxicidad de plaguicidas y su asociación con la enfermedad de Parkinson. Arch Neurocien. 2011;16:33-9.

10. Ortiz GG, Pacheco-Moisés FP, Mireles-Ramírez MA et al Oxidative stress and Parkinson's disease: effects on environmental toxicology. En: Ahmad R, editor. Free radicals and diseases. Rijeka: InTech; 2016. p. 139-151.

11. Acuña-Castroviejo D, Coto-Montes A, Gaia Monti M, et al. Melatonin is protective against MPTP-induced striatal and hippocampal lesions. Life Sci. 1997;60:PL23-9.
12. Ortiz GG, Reiter RJ, Zúñiga G, et al. Genotoxicity of paraquat: micronuclei induced in bone marrow and peripheral blood are inhibited by melatonin. Mutat Res. 2000;464:239-45

13. Ortiz GG, Pacheco-Moisés FP, Gómez-Rodríguez VM, et al. Fish oil, melatonin and vitamin $\mathrm{E}$ attenuates midbrain cyclooxygenase-2 activity and oxidative stress after the administration of 1-methyl-4-phenyl-1,2,3,6- tetrahydropyridine. Metab Brain Dis. 2013;28:705-9.

14. Ortiz GG, Crespo-López ME, Morán-Moguel C, et al. Protective role of melatonin against MPTP-induced mouse brain cell DNA fragmentation and apoptosis in vivo. Neuro Endocrinol Lett. 2001;22:101-8.

15. Pandi-Perumal SR, BaHammam AS, Brown GM, et al. Melatonin antioxidative defense: therapeutical implications for aging and neurodegenerative processes. Neurotox Res. 2013;23:267-300.

16. Cardinali DP, Esquifino Al, Srinivasan V, et al. Melatonin and the immune system in aging. Neuroimmunomodulation. 2008;15:272-8.

17. Tan D-X, Manchester LC, Terron MP, et al. One molecule, many derivatives: a never-ending interaction of melatonin with reactive oxygen and nitrogen species? J Pineal Res. 2007;42:28-42.

18. Galano A, Tan DX, Reiter RJ. Melatonin as a natural ally against oxidative stress: a physicochemical examination. J Pineal Res. 2011; 51:1-16.

19. Ortiz GG, Benítez-King G, Rosales-Corral S, et al. Cellular and biochemical actions of melatonin which protect against free radicals: role in neurodegenerative disorders. Curr Neuropharmacol. 2008;6:203-14.

20. Rothman SM, Mattson MP. Sleep disturbances in Alzheimer's and Parkinson's diseases. Neuromolecular Med. 2012;14:194-204.

21. Srinivasan V, Cardinali DP, Srinivasan US, et al. Therapeutic potential of melatonin and its analogs in Parkinson's disease: focus on sleep and neuroprotection. Ther Adv Neurol Disord. 2011;4:297-317.

22. Minghetti L. Role of COX-2 in inflammatory and degenerative brain diseases. Subcell Biochem. 2007;42:127-41.

23. Benitez-King G. Melatonin as a cytoskeletal modulator: implications for cell physiology and disease. J Pineal Res. 2006;40:1-9.

24. Wiesenberg I, Missbach M, Carlberg C. The potential role of the transcription factor RZR/ROR as a mediator of nuclear melatonin signaling. Restor Neurol Neurosci. 1998;12:143-50.

25. Hardeland R, Cardinali DP, Srinivasan V, et al. Melatonin - a pleiotropic, orchestrating regulator molecule. Prog Neurobiol. 2011;93:350-84. 